Ann. Biol. anim. Bioch. Biophys., 1977, 17 (4), 503-508.

\title{
Motricité cæcale et transit chez le poney : Influence de l'état de réplétion du cæcum et des fermentations microbiennes
}

\author{
par M. CANDAU, L. BUENO *
}

\author{
Ecole Nationale Supérieure Agronomique \\ 145, avenue de Muret, 31300 Toulouse Cedex \\ * Ecole Nationale Vétérinaire \\ 23, chemin des Capelles, 31076 Toulouse Cedex.
}

Summary. Caecal motility and digesta transit rate in the pony. Effects of volume of caecal contents and of microbial digestion.

The basic motility of the cecum recorded during 4 to 6 weeks on three ponies showed two patterns : large contractions moving the entire surface of the organ and smaller local contractions. The former greatly increased during feeding and appeared to move the ingesta towards the large intestine ; the latter varied with the end-products of digestion at this level. Electrical spiking activity of the caecum increased eight-fold and transit rate increased four-fold when the volume of caecal contents doubled. The role of caecal motility in regulating the volume of caecal contents is discussed.

Les phénomènes moteurs du tractus digestif des équidés sont mal connus chez le sujet éveillé en raison des difficultés chirurgicales y compris pour le cæcum (Howell et Cupp, 1950 ; Barcus, 1962) dont la fistulation est cependant la plus aisée (Lowe, Hintz et Schryver, 1970). Trois types différenciés de mouvements : grandes et petites contractions, variations de tonus, ont été décrits par Howell et Cupp. La nature des contractions en fonction de l'activité alimentaire et de l'état de réplétion du cæcum reste cependant à élucider.

Par ailleurs, des modifications de la durée totale du transit alimentaire de 26 à $37 \mathrm{~h}$ selon que le foin est broyé ou long (Wolter, Durix et Letourneau, 1974) et des variations de 12 à $48 \mathrm{~h}$ du temps de rétention de la phase solide du contenu digestif (Argenzio at al., 1974) sont en faveur d'un rôle important de l'activité motrice du cæcum dans la régulation du transit des digesta.

Ce travail a envisagé, d'une part, par le biais du l'électromyographie, les phénomènes moteurs du cæcum chez le poney en vue de préciser les facteurs responsables de son activité motrice, d'autre part, les variations du débit cæco-colique en fonction du degré de réplétion de l'organe ef de sa motricité en vue d'apprécier son rôle dans le transit. 


\section{Matériel et Méthodes.}

Enregistrement des phénomènes moteurs.

Trois poneys Pottock adultes de $200 \mathrm{~kg}$ ont été munis d'une canule du cæcum en vue de l'étude des variations de la pression endoluminale par la méthode des ballonnets. Des groupes de trois électrodes de réception intrapariétales ont été fixés à 10 ou $15 \mathrm{~cm}$ d'intervalle selon la technique de Ruckebusch (1973) sur la paroi du cæcum ainsi que sur celle de l'iléon terminal et du côlon replié.

L'implantation intrapariétale de deux microballonnets (diam. : $4 \mathrm{~mm}$ ) à proximité de deux groupes d'électrodes a été réalisée en vue de l'analyse des variations de la tension musculaire locale.

Les animaux ont reçu du foin ad libifum ou à heures fixes. Des périodes de jeûne alimentaire de $24 \mathrm{~h}$ ont été imposées à trois reprises. L'état de réplétion du cæcum a été modifié pendant le repas par soustraction du contenu cæcal (7 à $10 \mathrm{l}$ ) en 60-90 mn à l'aide d'une pompe vide, suivie le lendemain, de l'introduction du contenu à $37^{\circ} \mathrm{C}$ en milieu anaérobie.

Des perfusions intracæcales de solutions ammoniacales $(0,1 \mathrm{~mole} / \mathrm{l} / \mathrm{h})$ à $\mathrm{pH}: 7,5$, de solutions d'acides gras volatils $(0,5 \mathrm{~mole} / \mathrm{l} / \mathrm{h})$ à $\mathrm{pH}: 6,6$ contenant les acides acétique $(60$ p. 100$)$, propionique $(30$ p. 100$)$ et butyrique $(10$ p. 100) ont été réalisées pendant $2 \mathrm{~h}$.

Mesure du débit cæco-colique.

Le débit cæco-colique a été mesuré sur trois poneys Pottock à l'aide de PEG 4000 $(20 \mathrm{~g})$ introduit par la canule du cæcum. Une nouvelle addition de PEG $4000(20 \mathrm{~g})$ permet de calculer au bout de $5 \mathrm{~h}$ le volume final (Hyden, 1955a, b). La valeur du débit est obtenue en dérivant la fonction exponentielle des concentrations en fonction du temps (Bueno, 1972).

\section{Résultats.}

Types de motricité du cæcum.

La figure 1 montre l'existence de contractions propagées dans les deux sens entre la base et la pointe, la vitesse de propagation étant plus élevée dans le sens basepointe (12 au lieu de $10 \mathrm{~cm} / \mathrm{s}$ ). Les salves correspondant aux contractions, qui peuvent être simples ou groupées, durent 3 à $6 \mathrm{~s}$, l'amplitude des potentiels étant comprise entre 100 et $200 \mu \mathrm{v}$. La pression développée au niveau des microballonnets est de l'ordre de 10-20 $\mathrm{mmHg}$.

On relève également la présence, surtout au niveau de la pointe de l’organe, de contractions localisées et sans rythmicité bien définie. Celles-ci sont groupées par phases de trois à huit minutes ef la durée des salves de potentiels correspondantes est de 1 à $2 \mathrm{~s}$ avec une amplitude de $100 \mu \mathrm{v}$. La pression développée est toujours inférieure à $10 \mathrm{~mm} \mathrm{Hg}$. 
Certaines salves de potentiels (1-2 s, $100 \mu v)$ apparaissent également de façon aléatoire et isolée. Cette activité qualifiée de dispersée parce que ni propagée, ni localisée, est importante.

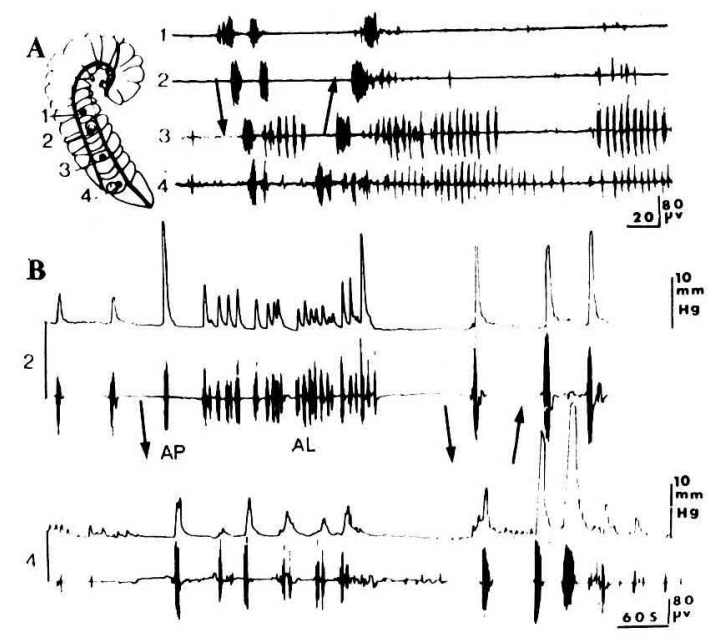

FIG. 1. - Types de motricité du cæcum. En A, électromyogrammes recueillis à partir de groupes d'électrodes (•) distants de $10 \mathrm{~cm}$ entre $1-2$ et de $15 \mathrm{~cm}$ entre 2-3 et 3-4. Les flèches indiquent le sens de la propagation des contractions. En $B$, mécanogrammes correspondant à des microballonnets (o) placés à proximité des groupes d'électrodes 2 et 4 . La pression développée pour les contractions propagées (AP) est voisine de $20 \mathrm{~mm} \mathrm{Hg}$. Elle est inférieure à $10 \mathrm{~mm} \mathrm{Hg}$ pour les contractions localisées (AL).

Au cours du jeûne, la motricité globale est à peu près constante et de faible intensité. Chez le sujet nourri ad libitum la motricité cæcale est accrue durant la totalité du nycthémère, l'augmentation de l'activité correspondant toujours à une période de prise de nourriture. L'augmentation de l'activité propagée cesse dès la fin du repas alors que l'activité localisée reste élevée durant une à deux heures (tabl. 1).

\section{TABLEAU 1}

Influence de l'activité alimentaire sur lo motricité cæcale chez un même sujet laissé à jeân pendant $24 \mathrm{~h}$, nourri ad libitum ou recevant un seul repas de foin de deux $h$ (valeurs moyennes ef écarts-types pour trois essais).

Types de motricité

Activité propagée (contractions par $10 \mathrm{mn}$ ) $\ldots \ldots \ldots \ldots \ldots \quad 1,5 \pm 0,1$

Activité localisée (p. 100 du temps d'enregistrement) . . . . 19,4 $\overrightarrow{ \pm}, 1$
Ad libitum

$(24 \mathrm{~h})$

$8,9 \pm 3,910,3 \pm 2,0\left(^{*}\right)$

Valeurs en dehors du repas : $\left(^{*}\right) 5,6 \pm 2,3,\left(^{* *}\right) 21,6 \pm 5,5$ 
Effets de l'état de réplétion du cæcum.

La vidange du cæcum au cours d'un repas diminue, pendant 3 à 4 h, l'activité propagée et réduit fortement durant 5 à $7 \mathrm{~h}$ l'activité localisée (fig. $2 \mathrm{~A}$ ). L'introduction dans le cæcum, pendant un repas, de 10-12 I de contenu double l'activité propagée. L'activité localisée est également accrue durant plusieurs heures (fig. 2 B). La distension d'un ballonnet dans le cæcum provoque une réponse contractile de type propagé sans modifier l'activiłé localisée.

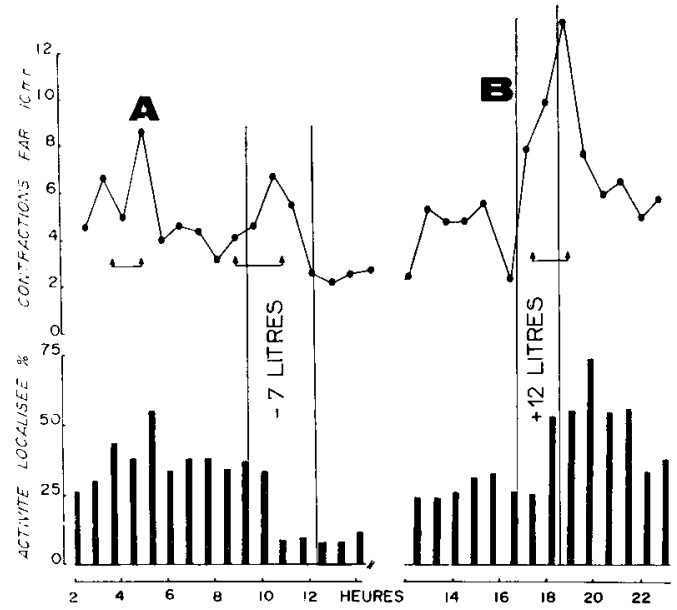

FIG. 2. - Etat de répléfion du cæcum et motricité. Effets du prélèvement de 7 I de contenu (A) et de la perfusion de 12 I de jus cæcal (B) par rapport à l'ingestion d'un repas de foin.

A noter enfin que l'activité iléale, accrue pendant les repas, n'est pas modifiée par les variations de l'état de réplétion du cæcum. En revanche, l'activité colique est plus. ou moins intense en relation avec la motricité du cæcum de type propagée, y compris; dans le cas de perfusion ; en outre, chaque contraction dans le sens pointe-base du; cæcum se prolonge sur le côlon replié dorsal.

\section{Effets de l'administration de métabolites cæcaux.}

Les perfusions de $\mathrm{NH}_{3}$ et d'AGV augmentent la fréquence des contractions propagées durant une heure environ et inhibent ensuite ce type d'activité. Un même volume d'eau à $37^{\circ} \mathrm{C}$. accroît également la fréquence des contractions propagées durant le même temps. Une phase prolongée d'hypermotricité localisée apparaît de façon spécifique pour les $\mathrm{AGV}$ et $\mathrm{NH}_{3}$. La réponse est plus intense à la base qu'à la pointe pour les deux substances, l'effet des AGV étant supérieur à 50 p. 100 environ à celui de $\mathrm{NH}_{3}$. La figure 3 montre que l'hypercinésie localisée en réponse aux AGV est à la fois plus intense et plus précoce que celle due à $\mathrm{NH}_{3}$. En outre, la réponse à $\mathrm{NH}_{3}$ semble complexe à en juger par l'allure biphasique de l'effet excito-moteur. 
Débit cæco-colique, volume cæcal ef motricité cæcale.

Le volume du contenu cæcal reste relativement constant pendant les périodes de mesure du débif cæeco-colique (tabl. 2). Sa valeur est minimum, pour le régime

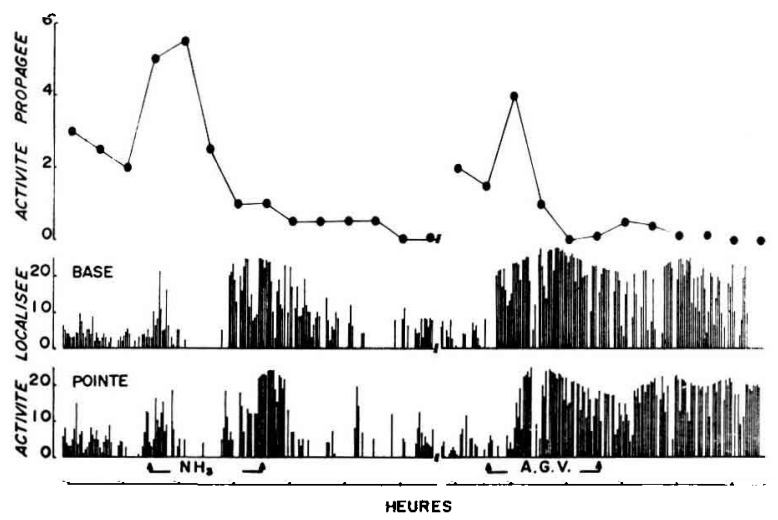

FIG. 3. - Motricité cæcale ef méłabolites digestifs. Effet prolongé sur l'activité localisée et bref sur l'activité propagée d'une perfusion intracxecale d'ammoniaque $(\mathrm{pH} 7,5,0,1 \mathrm{~mole} / \mathrm{l} / \mathrm{h})$ et d'un mélange d'acides gras volatils ( $\mathrm{pH} 6,6,0,5 \mathrm{~mole} / \mathrm{l} / \mathrm{h}$ ).

foin-granulés. Le volume du contenu cæcal et le débit cæco-colique sont réduits de plus de moitié après une période de jeûne de $24 \mathrm{~h}$, quel que soit le régime. Les valeurs obtenues montrent qu'à de faibles variations du volume cæcal correspondent des accroissements importants du débit cæco-colique. L'examen de l'activité motrice du cæcum ef de la vitesse de disparition d'un traceur montre que les variations de motri-

TABLEAU 2

Débit iléo-cæcal et motricité cæcale chez trois poneys en fonction des modalités alimentaires (moyenne et écart-type pour trois mesures individuelles)

\begin{tabular}{|c|c|c|c|c|}
\hline \multirow{2}{*}{$\frac{\text { Régime alimentaire }}{\text { Conditions expérimentales }}$} & \multicolumn{2}{|c|}{ Foin } & \multicolumn{2}{|c|}{ Foin et granulés } \\
\hline & Ad libifum* & $\begin{array}{l}\text { A jeôn } \\
(24 \mathrm{~h})\end{array}$ & Ad libitum * & $\begin{array}{l}\text { A jeûn } \\
(24 h)\end{array}$ \\
\hline \multicolumn{5}{|l|}{ Volume cæcal (I) } \\
\hline Initial .......... & $10,2 \pm 1,1$ & $6,6 \pm 1,2$ & $8,1 \pm 0,9$ & $6,1 \pm 0,8$ \\
\hline $\begin{array}{c}\text { Final } \ldots \ldots \ldots \\
\text { Débit cxco-colique }\end{array}$ & $11,9 \pm 0,9$ & $5,8 \pm 0,9$ & $6,1 \pm 0,8$ & $6,3 \pm 0,9$ \\
\hline $\begin{array}{l}\text { Débit crco-colique } \\
(\mathrm{l} / \mathrm{h}) \ldots \ldots \ldots \ldots \ldots\end{array}$ & $37+04$ & $1,0+0,2$ & $3,6+0,5$ & $0,9 \pm 0,1$ \\
\hline $\begin{array}{l}\text { Motricité cxcale } \\
\text { (p. } 100 \text { d'activité durant } 7 \text { h)........ }\end{array}$ & & & & \\
\hline (p. 100 d'activité durant 7 h)........ & $53,4 \pm 4,3$ & $6,6 \pm 2,7$ & $52,7 \pm 4,2$ & $6,1 \pm 3,5$ \\
\hline
\end{tabular}

* Valeurs lorsque l'animal n'a pas accès aux aliments pendant les mesures :

$8,1 \pm 1,0 \mathrm{l}, 2,4 \pm 0,6 \mathrm{l} / \mathrm{h}, 30,5 \pm 4,6 \mathrm{p} .100$ pour le régime foin ;

$7,3 \pm 0,91,2,3 \pm 0,9 \mathrm{l} / \mathrm{h}, 28,4 \pm 5,2 \mathrm{p} .100$ pour le régime foin-granulés. 
cité sont relativement beaucoup plus importantes que celles du débit. On retrouve entre ces deux éléments une liaison de type exponentielle analogue à celle existant entre volume ef débit.

\section{Discussion et conclusion.}

L'étude électromyographique de la motricité cæcale confirme l'existence de deux types majeurs de contractions, les unes propagées, les autres localisées. L'influence de l'état de réplétion du cæcum sur la motricité propagée est manifeste puisqu'elle disparaît après prélèvement du contenu cæcal et qu'elle est renforcée par la perfusion d'un volume égal à la moitié environ du contenu cæacal. Les contractions propagées ont soit un rôle de réplétion (suggéré par l'existence au niveau de l'iléon d'une activité péristaltique précédant la mise en tension du cæcum dans le sens basepointe), soit un rôle de vidange (suggéré par une activité colique et péristaltique consécutive à la propagation dans le sens pointe-base).

La résultante de ces deux effets opposés est un ajustement remarquable du volume du contenu cæcal autour d'une valeur constante. Les mécanismes mis en jeu apparaissent particulièrement efficaces. L'importance de la riposte motrice à de faibles variations volumétriques enregistrées chez le sujet à jeûn ou nourri à volonté est dans le rapport de 1 à 8 entraînant des variations dans le rapport de 1 à 4 du débit cæco-colique. Le cæcum apparaît donc comme un organe de régulation du transit des digesta entre l'intestin ef le côlon.

L'activité localisée est liée à la stimulation chimique par les AGV ef $\mathrm{NH}_{3}$, l'existence d'une chémo-sensibilité facilitant l'absorption par le biais d'une motricité accrue est probable.

Commission CNERNA Digestion-Absorption, Tours, 13 novembre 1976.

\section{Références}

ARGENZIO J. B., LOWE J. E., PICKARD D. W., STEVENS C. E., 1974. Digesta passage and water exchange in the equine large intestine. Am. J. Physiol., 226, 1035-1042.

BARCUS B., 1962. Recherches sur la motricité normale du cæcum des équidés. Thèse Doct. Vét. Toulouse, $84 \mathrm{p}$.

BUENO L., 1972. Etudes des mouvements hydriques au niveau du feuillet chez le mouton. Ann. Rech. vét., 3, 651-663.

HOWELL C. E., CUPP P. T., 1950. Motility patterns of the creum of horse. J. anim. Sci., 9, 261-268.

HYDEN S., 1955a. Turbidimetric method for the determination of higher polyethylene glycols in biological materials. K. Lantbrhögsk. Annir., 22, 139-145.

HYDEN S., 1955b. The recovery of polyethylene glycols after passage through the digestive tract. K. Lantbrhögsk. Annlr., 22, 411-424.

LOWE J. E., HINTZ H. F., SCHRYVER J. F., 1970. A new technique for long-term caecal fistulation in ponies. Am. J. vet. Res., 31, 6, 1109-1111.

RUCKEBUSCH Y., 1973. L'électromyographie globale des muscles lisses à partir d'électrodes chroniques intra pariétales souples. Rev. Méd. vét., 124, 1407-1434.

WOLTER R., DURIX A., LETOURNEAU J. C., 1974. Influence du mode de présentation du fourrage sur la vitesse du transit digestif chez le poney. Ann. Zootech., 23, 293-300. 